\title{
AVALIAÇÃO DA CARCAÇA DE CORDEIROS DA RAÇA TEXEL SOB DIFERENTES MÉTODOS DE ALIMENTAÇÃO E PESOS DE ABATE ${ }^{1}$
}

\author{
CARCASS EVALUATION OF TEXEL LAMBS, UNDER DIFFERENT FEEDING \\ SYSTEMS AND SLAUGHTER WEIGHTS
}

\author{
Otacílio Silva da Motta ${ }^{2}$ Cleber Cassol Pires ${ }^{3}$ José Henrique Souza da Silva ${ }^{4}$ \\ Gilberto Teixeira da Rosa ${ }^{5}$ Márcio Fülber ${ }^{6}$
}

\section{RESUMO}

O trabalho objetivou avaliar o efeito do sexo, peso de abate e métodos de alimentação sobre as características qualitativas e quantitativas da carcaça de cordeiro (as) da raça Texel. O experimento utilizou 38 animais $(20$ cordeiros machos não castrados e 18 cordeiras), os quais foram aleatoriamente distribuídos, 24 horas após o nascimento, com suas respectivas mães, em três métodos de alimentação $(M 1=$ ovelhas alimentadas com silagem de milho, cordeiros com acesso a "creep feeding" $e$ desmamados aos 60 dias de idade; $M 2=$ ovelhas alimentadas com silagem de milho, cordeiros com acesso ao "creep feeding" $e$ desmamados aos 45 dias; M3= ovelhas alimentadas com silagem de milho e concentrado e cordeiros desmamados aos 60 dias). Os cordeiros(as) em cada método e sexo foram abatidos com 25 ou $33 \mathrm{~kg}$ de peso vivo. Houve influência significativa $(P<0,05)$ do método de alimentação e peso de abate sobre o peso de carcaça, não ocorrendo entre sexo. Ocorreu interação significativa $(P<0,05)$ entre métodos de alimentação, sexo e peso de abate para a variável rendimento de carcaça quente, e quanto as características quantitativas da carcaça, o comprimento de carcaça demonstrou diferença significativa $(P<0,05)$ entre os métodos de alimentação, sendo que a área de lombo, comprimento de carcaça, comprimento de perna e espessura de coxão foram significativamente $(P<0,05)$ mais pesados a um maior peso ao abate, sem que houvesse diferença significativa $(P>\geq 0,05)$ entre sexo. $O$ método de alimentação não influenciou significativamente $(P \geq 0,05)$ a espessura de gordura, marmoreio $e$ a gordura de cobertura, no entanto estas variáveis foram influenciadas a $(P<0,05)$ pelo sexo e peso ao abate, sendo que para gordura de cobertura não houve diferença significativa $(P \geq 0,05)$ nos diferentes pesos. Os resultados mostram que um alto rendimento de carcaça de cordeiros machos não castrados abatidos aos $25 \mathrm{~kg}$ é obtido quando os mesmos são submetidos a um alto nível nutricional no período de cria e terminação.

Palavras-chave: “creep feeding”, desmame, rendimento de carcaça, silagem de milho.

\section{SUMMARY}

The objetive of this work was to evaluate the effect of sex, slaughter weight and feeding method in qualitative and quantitative characteristcs of lambs (males and female) of Texel breeds. The experiment was conducted, using 38 animals $(20$ males not castrated and 18 females ).randonly distribuited, 24 hours after birth, with their mothers, in three feeding systems (M1-females fed with corn silage, lambs having acess to creep feeding and weaned at 60 days of age; M2-females fed with corn silage, lambs having access to creep feeding and weaned at 45 days of age; M3-females fed with corn silage and concentrated and lambs weaned at 60 days of age). The lambs (males and females) in each method and Sex were slaughtered with 25 or $33 \mathrm{~kg}$ of live weight. There was a significant influence $(P<0.5)$ of feeding method and slaughter weight in the carcass weight, but not of sex. There was a significant interaction $(P<0.5)$ among feeding methods, sex and slaughter weight in the yield of warm carcass. Related to the quantitative carcass characteristcs, the carcass length differed significantly $(P<0.5)$ for the feeding methods, having bigger back area, carcass length and thigh thickness at higher slaughter weight, without significant difference $(P \geq 0.5)$ between sexes. The feeding method was not significant $(P \geq 0.5)$ for fat thickness, marbling and fatness cover; however, those characteristcs were influenced $(P<0.5)$ by sex and

\footnotetext{
${ }^{1}$ Parte da Dissertação de Mestrado do primeiro autor, apresentada ao Departamento de Zootecnia (DZ), Universidade Federal de Santa Maria (UFSM)

${ }^{2}$ Médico Veterinário, Professor da EAFA

${ }^{3}$ Professor do departamento de Zootecnia, UFSM, Camobi, 97105-900, Santa Maria, RS. E-mail: cpires@ccr.ufsm.br. Autor para correspondência.

${ }^{4}$ Professor do departamento de Zootecnia, UFSM

${ }^{5}$ Aluno de Pós-graduação da UFSM.

${ }^{6}$ Acadêmico da graduação em Veterinária da UFSM.
} 
slaughter weight, being the fatness cover not significant at different weights. The results showed that a best income of not castred male lambs carcasses slaughtered with $25 \mathrm{~kg}$, is obtained when they are submited to a good nutrition level during the growing and finishing periods.

Key words: “creep feeding”, weaning, carcass yeld, corn silage.

\section{INTRODUÇÃO}

A criação de ovinos com o propósito carne surge como uma boa alternativa para as propriedades rurais em diversas regiões brasileiras. SIQUEIRA (1983) cita que idades muito avançadas dos animais ou dietas que propiciem elevada deposição de gordura na carcaça devem ser evitadas, sendo isso um ponto fundamental para o consumidor.

Atualmente um dos aspectos importantes a se considerar no mercado de carne é o conteúdo de gordura, em função da sua correlação com problemas de saúde humana. Segundo SAINZ (1996), na espécie ovina a gordura é o componente de maior variabilidade na carcaça, estando a espessura de gordura associada a vários fatores, entre eles, a raça do animal, sexo, regime alimentar, duração do período alimentar e o peso da carcaça. SANTOS (1999) afirma que a eficiência na produção de carne, com máximo de músculo e adequada quantidade de gordura, é o objetivo dos sistemas modernos de produção.

A tipificação é necessária para garantir a qualidade da carcaça de cordeiro(as) objetivando oferecer confiança permanente ao consumidor. Para isso, o sexo do animal deve ser considerado pelo produtor. DEAMBROSIS (1972) e SAINZ (1996) citam que o conteúdo de gordura é menor no macho não castrado, seguido pelo castrado e, por último, a fêmea. Todas as técnicas desenvolvidas na criação, manejo e melhoramento animal, tem por objetivo único a obtenção de uma boa carcaça dentro de um menor tempo, sendo que a qualidade do produto e quantidade da parte comestível são os fatores básicos do mérito da carcaça (SALOMONI,1981).

O objetivo deste trabalho foi avaliar as principais características qualitativas e quantitativas da carcaça de cordeiros machos não castrados e fêmeas em três métodos de alimentação e abatidos a dois pesos, 25 ou $33 \mathrm{~kg}$.

\section{MATERIAL E MÉTODOS}

O experimento foi conduzido no setor de ovinocultura do departamento de zootecnia da Universidade Federal de Santa Maria, no período de julho de 1998 a janeiro de 1999. Foram utilizados 38 cordeiros (20 machos não castrados e 18 fêmeas), oriundos de um cruzamento de absorção com a raça Texel que, 24 horas após o nascimento, foram distribuídos aleatoriamente, com suas respectivas mães, em três métodos de alimentação; Método 1 (M1)= Alimentação constituída de silagem de milho para o conjunto (ovelha + cordeiro) e concentrado apenas aos cordeiros (as) na forma de "creep feeding" do nascimento ao desmame (60 dias); Método 2 (M2)= Alimentação constituída de silagem de milho para o conjunto (ovelha + cordeiro) e concentrado apenas aos cordeiros (as) na forma de "creep" do nascimento ao desmame (45 dias); Método 3 (M3)= Alimentação constituída de silagem de milho + concentrado para o conjunto (ovelha + cordeiro), do nascimento ao desmame (60 dias). Cada método foi constituído de dois sexos e os cordeiros(as) abatidos com 25 e $33 \mathrm{~kg}$.

Os animais foram mantidos em galpão de alvenaria coberto com telha de amianto e, em cada método, foram alimentados em baias coletivas com piso de cimento. Utilizou-se, para os animais do M1 e M2, uma dieta constituída de silagem de milho, contendo 8,08\% de Proteína Bruta (PB) e 66,04\% de Nutrientes Digestíveis Totais (NDT), sendo que os cordeiros desses métodos receberam através do "creep" um concentrado com $20 \%$ de PB e $77 \%$ de NDT na quantidade de $2 \%$ do peso vivo. Para o M3, foi fornecida uma dieta para atender as exigências nutricionais das ovelhas em lactação, constituída por silagem de milho + concentrado na proporção de volumoso/concentrado de 70:30 na Matéria Seca (MS), contendo $13,5 \%$ de PB e $65 \%$ de NDT, conforme o NRC (1985). Após o desmame, cada lote de cordeiros recebeu uma dieta de silagem de milho + concentrado na proporção de volumoso/concentrado de 50:50 na MS contendo $16,7 \%$ de PB e $80 \%$ de NDT.

As pesagens dos animais foram realizadas ao início do período experimental, 24 horas após o nascimento, ao desmame e imediatamente antes do abate, além destas foram realizadas pesagens intermediárias de 21 em 21 dias. As pesagens ao desmame e antes do abate final foram realizadas pela manhã posterior ao jejum de sólidos por 14 horas. Os animais, independente de sexo, foram abatidos com idade média de 133,67 dias (M1), 133,04 dias (M2) e 110 dias (M3). Para os pesos de abate de $25 \mathrm{~kg}$ e de $33 \mathrm{~kg}$, foram de 105,13 e de 125,44 dias respectivamente, independente de sexo e métodos.

Após o abate, as carcaças foram imediatamente pesadas e colocadas em câmara fria por 24 horas a $2^{\circ} \mathrm{C}$ e novamente pesadas, e calculado 
o índice de quebra ao resfriamento. Nesse momento foram tomadas as medidas objetivas e subjetivas das carcaças. A área de lombo foi obtida pela exposição do músculo Longissimus dorsi, após um corte transversal na carcaça, entre a $12^{\mathrm{a}}$ e $13^{\mathrm{a}}$ costela, traçando o seu contorno em papel vegetal e para determinação e registro da área, foi utilizado o programa SITER 2.2 modelo A 2. O comprimento interno da carcaça (distância entre o bordo anterior da sínfise ísquio-pubiana e o bordo anterior medial da $1^{a}$ costela), o comprimento de perna (distância entre o bordo anterior da sínfise ísquio-pubiana e a porção média dos ossos do tarso) e a espessura de coxão (distância entre a face medial interna e externa da parte superior da perna em sua parte mais larga) foram obtidas com um compasso de madeira com pontas metálicas, e a espessura de gordura de cobertura foi determinada em um ponto central na porção média do músculo Longíssimus dorsi exposto na sua porção distal, entre a $12^{\underline{a}}$ e $13^{\underline{a}}$ costela. O marmoreio foi avaliado subjetivamente através da existência de depósitos de gordura entre as fibras musculares no Longíssimus dorsi, conforme índices crescentes variando de 1 (inexistente) a 5 (excessivo). A gordura de cobertura foi avaliada subjetivamente pela quantidade e distribuição da gordura externa na carcaça, através de índices crescentes variando de 1 (magra) a 5 (muito gorda), adaptados do sistema proposto por MÜLLER (1987) e OSÓRIO (1998).

Os dados de peso e características da carcaça foram submetidos à análise de variância, aplicando-se o teste $\mathrm{F}$ ao nível de $5 \%$ e as médias comparadas pelo teste Pdiff ao mesmo nível de significância. As análises estatísticas foram realizadas utilizando-se o pacote estatístico SAS (1996) versão 6.

\section{RESULTADOS E DISCUSSÃO}

$\mathrm{Na}$ tabela 1, são apresentados os valores médios de peso de carcaça quente, peso de carcaça fria e índice de quebra ao resfriamento. Não houve significância $(\mathrm{P} \geq 0,05)$ para as interações entre os métodos de alimentação, sexo e pesos de abate; sendo assim, os resultados são apresentados de forma independente. Houve efeito do método de alimentação sobre os pesos de carcaça quente e fria. Este fato é mais evidente quanto ao peso de carcaça fria pois ao melhor nível nutricional (M3) correspondeu ao maior peso de carcaça. Isto ocorreu em função do maior desenvolvimento de massa muscular já que o índice de quebra (Tabela 1), espessura e gordura de cobertura (Tabela 2) não diferiram entre métodos. SAINZ (1996) cita que o
Tabela 1 - Médias e erros padrão para o peso de carcaça quente (PCQ), peso de carcaça fria (PCF) e índice de quebra ao resfriamento (IQR) de acordo com os métodos de alimentação (M1, M2 e M3), sexo (machos não castrados e fêmeas) e pesos de abate $(25$ e $33 \mathrm{~kg})$.

\begin{tabular}{cccc}
\hline Fatores/Níveis & $\mathrm{PCQ}(\mathrm{kg})$ & $\mathrm{PCF}(\mathrm{kg})$ & $\mathrm{IQR}(\%)$ \\
\hline M1 & $13,35^{\mathrm{a}} \pm 0,188$ & $12,91^{\mathrm{b}} \pm 0,186$ & $4,20^{\mathrm{a}} \pm 0,848$ \\
M2 & $13,07^{\mathrm{b}} \pm 0,182$ & $12,66^{\mathrm{b}} \pm 0,180$ & $5,75^{\mathrm{a}} \pm 0,821$ \\
M3 & $13,75^{\mathrm{a}} \pm 0,182$ & $13,48^{\mathrm{a}} \pm 0,180$ & $4,99^{\mathrm{a}} \pm 0,821$ \\
F & 3,58 & 3,66 & 0,86 \\
$\mathrm{P}$ & 0,042 & 0,039 & 0,434 \\
Machos & $13,41^{\mathrm{a}} \pm 0,147$ & $12,99^{\mathrm{a}} \pm 0,145$ & $4,86^{\mathrm{a}} \pm 0,663$ \\
Fêmeas & $13,37^{\mathrm{a}} \pm 0,153$ & $12,96^{\mathrm{a}} \pm 0,151$ & $5,10^{\mathrm{a}} \pm 0,692$ \\
F & 0,04 & 0,02 & 0,06 \\
$\mathrm{P}$ & 0,85 & 0,88 & 0,80 \\
25 & $11,41^{\mathrm{b}} \pm 0,147$ & $11,00^{\mathrm{b}} \pm 0,145$ & $5,66^{\mathrm{a}} \pm 0,663$ \\
33 & $15,37^{\mathrm{a}} \pm 0,153$ & $14,94^{\mathrm{a}} \pm 0,151$ & $4,29^{\mathrm{a}} \pm 0,692$ \\
F & 346,05 & 349,71 & 2,03 \\
$\mathrm{P}$ & 0,0001 & 0,0001 & 0,1658 \\
Média & 13,29 & 12,88 & 5,02 \\
CV(\%) & 4,9 & 5,0 & 58,52 \\
\hline
\end{tabular}

Médias seguidas de letras diferentes na coluna, em cada fator, diferem $(\mathrm{P}<0,05)$ entre si pelo Teste Pdiff.

peso da carcaça é influenciado pela velocidade de crescimento, idade ao abate e regime nutricional dos animais.

Em relação ao sexo, não houve diferença $(\mathrm{P}<0,05)$ entre os pesos de carcaça quente. CARVALHO (1998) também não encontrou diferença $(\mathrm{P} \geq 0,05)$ entre machos não castrados $(12,66 \mathrm{~kg})$, machos castrados $(12,60 \mathrm{~kg})$ e fêmeas $(11,97 \mathrm{~kg})$ ao trabalhar com cordeiros (as) da raça Texel, confinados e abatidos aos 100 dias de idade. $\mathrm{O}$ maior peso de carcaça correspondeu ao peso mais elevado de abate o que está de acordo com SOLOMON et al. (1980) que evidenciam que os animais abatidos com maior peso apresentam carcaças mais pesadas. $O$ índice de quebra ao resfriamento não mostrou diferença $(\mathrm{P} \geq 0,05)$ entre os métodos de alimentação, sexo e pesos de abate, o que pode ser devido ao alto coeficiente de variação encontrado. Mesmo assim, estes resultados estão de acordo com os de SIQUEIRA (1983) que não encontrou diferença $(P \geq 0,05)$ entre machos $5,26 \%$ e fêmeas $6,32 \%$, em cordeiros (as) da raça Ideal e Texel x Ideal. CARVALHO (1998) também não encontrou diferença $(\mathrm{P} \geq 0,05)$, obtendo para machos inteiros $3,05 \%$, para machos castrados $2,94 \%$ e para fêmeas $3,13 \%$.

Na tabela 3, são apresentados os valores médios de rendimento de carcaça quente dos cordeiros. A análise estatística dos dados referentes 
Tabela 2 - Médias e erros padrão para, área de lombo (AL) em centímetros quadrados, espessura de gordura (EG) em mm, marmoreio (M) com índice de 1 a 5, gordura de cobertura (GC) com índice de 1 a 5, de acordo com os métodos de alimentação (M1, M2 e M3), sexo (machos não castrados e fêmeas) e pesos de abate (25 e 33kg).

\begin{tabular}{ccccc}
\hline Fatores/Níveis & AL & EG & M & GC \\
\hline M1 & $10,66^{\mathrm{a}} \pm 0,466$ & $1,96^{\mathrm{a}} \pm 0,227$ & $3,04^{\mathrm{a}} \pm 0,268$ & $3,16^{\mathrm{a}} \pm 0,288$ \\
M2 & $10,87^{\mathrm{a}} \pm 0,451$ & $1,47^{\mathrm{a}} \pm 0,220$ & $2,75^{\mathrm{a}} \pm 0,245$ & $2,41^{\mathrm{a}} \pm 0,263$ \\
M3 & $11,30^{\mathrm{a}} \pm 0,466$ & $1,79^{\mathrm{a}} \pm 0,227$ & $2,75^{\mathrm{a}} \pm 0,253$ & $3,33^{\mathrm{a}} \pm 0,272$ \\
F & 0,48 & 1,24 & 0,41 & 3,32 \\
P & 0,63 & 0,306 & 0,660 & 0,053 \\
Machos & $11,08^{\mathrm{a}} \pm 0,373$ & $1,41^{\mathrm{b}} \pm 0,182$ & $2,36^{\mathrm{b}} \pm 0,211$ & $2,61^{\mathrm{b}} \pm 0,226$ \\
Fêmeas & $10,81^{\mathrm{a}} \pm 0,381$ & $2,07^{\mathrm{a}} \pm 0,186$ & $3,33^{\mathrm{a}} \pm 0,206$ & $3,33^{\mathrm{a}} \pm 0,222$ \\
F & 0,24 & 6,33 & 10,81 & 5,17 \\
P & 0,625 & 0,018 & 0,003 & 0,032 \\
25 & $9,96^{\mathrm{b}} \pm 0,373$ & $1,19^{\mathrm{b}} \pm 0,182$ & $2,25^{\mathrm{b}} \pm 0,211$ & $2,66^{\mathrm{a}} \pm 0,226$ \\
33 & $11,93^{\mathrm{a}} \pm 0,381$ & $2,29^{\mathrm{a}} \pm 0,186$ & $3,44^{\mathrm{a}} \pm 0,206$ & $3,27^{\mathrm{a}} \pm 0,222$ \\
F & 13,64 & 17,83 & 16,32 & 3,70 \\
P & 0,001 & 0,0003 & 0,0005 & 0,0660 \\
Média & 10,92 & 1,721 & 2,830 & 2,940 \\
CV(\%) & 14,79 & 45,87 & 30,98 & 32,01 \\
\hline
\end{tabular}

Médias seguidas de letras diferentes na coluna, em cada fator, diferem $(\mathrm{P}<0,05)$ entre si pelo Teste Pdiff.

à interação entre métodos de alimentação, sexo e pesos de abate foi significativa $(\mathrm{P}<0,05)$. $\mathrm{O}$ efeito do método de alimentação sobre o rendimento de carcaça, nos machos, foi verificado nos animais abatidos com $25 \mathrm{~kg}$, pois o maior valor obtido correspondeu ao melhor nível nutricional (M3). Nos machos abatidos aos $33 \mathrm{~kg}$ não houve diferenças entre métodos. Os cordeiros machos (M3) atingiram o peso de $25 \mathrm{~kg}$ com idade média de 78,25 dias, cerca de 18 dias após serem desmamados. O maior rendimento de carcaça dos machos (M3, abate aos $25 \mathrm{~kg}$ ), embora o pouco estado de engorduramento, está relacionado ao pouco desenvolvimento do trato gastrintestinal. Essa situação não ocorreu para o

Tabela 3 - Médias e erros padrão para rendimento de carcaça quente (RCQ) em percentual, de acordo com os métodos de alimentação (M1, M2 e M3), sexo (machos não castrados e fêmeas) e pesos de abate ( 25 e $33 \mathrm{~kg})$.

\begin{tabular}{|c|c|c|c|c|}
\hline \multirow[b]{3}{*}{ Métodos } & \multicolumn{4}{|l|}{ Sexo } \\
\hline & \multicolumn{2}{|c|}{ Machos } & \multicolumn{2}{|c|}{ Fêmeas } \\
\hline & 25 & 33 & 25 & 33 \\
\hline M1 & $45,11^{\mathrm{cd}} \pm 1,054$ & $47,28^{\mathrm{abc}} \pm 1,054$ & $44,71^{\mathrm{cd}} \pm 1,054$ & $45,98^{\mathrm{cd}} \pm 1,054$ \\
\hline M2 & $44,20^{\mathrm{d}} \pm 0,913$ & $45,59^{\mathrm{cd}} \pm 1,054$ & $45,18^{\mathrm{cd}} \pm 1,054$ & $46,71^{\text {bcd }} \pm 1,054$ \\
\hline M3 & $49,84^{\mathrm{a}} \pm 0,913$ & $46,13^{\mathrm{cd}} \pm 1,054$ & $44,82^{\mathrm{cd}} \pm 1,054$ & $49,28^{\mathrm{ab}} \pm 1,054$ \\
\hline
\end{tabular}

Médias seguidas de letras diferentes, diferem $(\mathrm{P}<0,05)$ entre sí pelo teste Pdiff. $\mathrm{F}=5,82 ; \mathrm{P}<0,0081 ;$ Média $=46,27 ; \mathrm{CV}(\%)=3,94$. abate dos machos aos $33 \mathrm{~kg}$, os quais foram abatidos com idade de 105,66 (M3), 131,33 (M1) e 139,33(M2).

Entre pesos de abate, os machos do M3 abatidos aos $33 \mathrm{~kg}$ obtiveram menor rendimento de carcaça quente que os abatidos aos $25 \mathrm{~kg}$. Isto pode ser devido ao aumento do conteúdo do trato gastrintestinal, sem que houvesse em contrapartida elevação da deposição de gordura na carcaça, que contribuiria para o aumento do peso e do rendimento da mesma. Sendo assim, o aumento do conteúdo digestivo elevou apenas o peso vivo ocasionando com isto, diminuição no rendimento de carcaça quente. SIQUEIRA \& FERNANDES, (1999) concluem que o conteúdo gastrintestinal pode promover, com as variações dos seus pesos, importantes oscilações no rendimento da carcaça. ROSA (2000), utilizando os mesmos animais do presente estudo, verificou que o conteúdo digestivo dos cordeiros abatidos aos $25 \mathrm{~kg}$ foi de $4,150 \mathrm{~kg}$ e dos abatidos aos $33 \mathrm{~kg}$ de $4,85 \mathrm{~kg}(\mathrm{P}<0,05)$.

$\mathrm{O}$ rendimento de carcaça das fêmeas do M3 abatidas aos $25 \mathrm{~kg}$ foram inferiores as do abate aos 33kg. Segundo ROSA (2000), o conteúdo digestivo não diferiu entre machos e fêmeas, portanto o maior valor obtido para as fêmeas aos $33 \mathrm{~kg}$, pode ser devido à maior deposição de gordura na carcaça. Esta afirmação está de acordo com SANTOS (1999) o qual observa que o rendimento de carcaça aumenta com a elevação do peso vivo e com o grau de acabamento do animal.

$\mathrm{Na}$ tabela 2, podem ser observados os valores médios da área de lombo, espessura de gordura, marmóreo e gordura de cobertura. As interações entre métodos de alimentação, sexo e pesos de abate não foram significativas $(P \geq 0,05)$, sendo assim, os resultados são apresentados de forma independente. $\mathrm{O}$ nível de alimentação e o sexo não afetaram significativamente $(\mathrm{P} \geq 0,05)$ a área de lombo. CARVALHO (1998) não obteve diferença significativa $(\mathrm{P} \geq 0,05)$ entre os cordeiros machos inteiros $11,21 \mathrm{~cm}^{2}$, machos castrados $11,38 \mathrm{~cm}^{2} \mathrm{e}$ fêmeas $11,23 \mathrm{~cm}^{2}$, confinados e abatidos com peso vivo de $27,09 \mathrm{~kg}$ aos 100 dias de idade. No entanto, para peso de abate houve diferença $(\mathrm{P}<0,05)$ sendo que os cordeiros (as) abatidos a um maior peso obtiveram maior área de lombo. HAMMOND (1959) e SAINZ (1996) 
referem que o músculo dorsal é de maturidade tardia, conseqüentemente ao abate mais tardio, o músculo teve um crescimento mais intenso.

O método de alimentação não determinou diferenças $(\mathrm{P} \geq 0,05)$ para espessura de gordura. SILVA (1986) também não encontrou diferença $(\mathrm{P} \geq 0,05)$ ao trabalhar com cordeiros da raça Ideal $(0,11 \mathrm{~cm})$ e para os cruzas Texel x Ideal $(0,08 \mathrm{~cm})$, criados em campo nativo. As fêmeas apresentam maior proporção de gordura na carcaça do que os machos não castrados $(\mathrm{P}<0,05)$. Esses resultados são concordantes com DEAMBRÓSIS (1972), o qual diz, que geralmente a proporção de gordura é menor nos machos inteiros, em relação a castrados e fêmeas. CARVALHO (1998) não encontrou diferença $(\mathrm{P} \geq 0,05)$ entre machos inteiros $(1,17 \mathrm{~cm})$, machos castrados $(2,17 \mathrm{~cm})$ e fêmeas $(2,00 \mathrm{~cm})$ em seu trabalho com cordeiros(as) da raça Texel, confinados e abatidos aos 100 dias. Entre pesos de abate, ocorreu diferença $(\mathrm{P}<0,05)$ sendo que a espessura de gordura se elevou com o aumento de peso. SENTS et al. (1982) relata que à medida que aumenta a idade e o peso de abate dos animais, a espessura de gordura se eleva.

Referente ao índice de marmoreio não se verificou diferença $(P \geq 0,05)$ entre os métodos de alimentação, no entanto, em relação ao sexo as fêmeas foram superiores, demostrando conforme OWEN (1976), que elas têm maturidade mais precoce, resultando numa maior deposição de gordura intramuscular. Quanto ao peso de abate houve diferença $(\mathrm{P}<0,05)$, com maior deposição de gordura no tecido intramuscular dos animais abatidos a um maior peso. Esse resultado está relacionado ao padrão de desenvolvimento da gordura que, embora classificada como de maturidade tardia, possui ordem de deposição de: gordura interna, intermuscular, sub-cutânea e intramuscular (KIRTON et al., 1972). BERG \& WALTERS (1983) comentam que o crescimento da gordura começa relativamente devagar e aumenta geometricamente quando os animais entram na fase de acabamento. Para gordura de cobertura, não ocorreu diferença $(\mathrm{P} \geq 0,05)$ entre os métodos de alimentação, sendo que o melhor nível de alimentação até o desmame não proporcionou maior acúmulo de gordura na carcaça, provavelmente por ser a gordura um tecido de desenvolvimento tardio comparado com músculo e osso, conforme BERG \& BUTERFIELD (1979). Sendo assim, não houve tempo necessário para que ocorresse deposição do tecido adiposo.
Em relação ao sexo, verifica-se diferença $(\mathrm{P}<0,05)$, evidenciando maior acúmulo de gordura, nas fêmeas pois estas apresentam uma maturidade fisiológica mais precoce. Para o peso de abate, não se obteve diferença $(\mathrm{P} \geq 0,05)$ na gordura de cobertura. No entanto, pode ser observado em valores numéricos uma elevação do menor para o maior peso, indicando que uma maior deposição de gordura de cobertura poderia ocorrer a um maior peso de abate.

\section{CONCLUSÕES}

O confinamento de ovelhas mais cordeiros e o livre acesso apenas dos cordeiros ao concentrado até o desmame aos 60 dias e confinamento destes até o abate não se constitui em vantagem ao desmame aos 45 dias e mesmo sistema, uma vez que este último também permite a obtenção de carcaças com pesos em torno de $13 \mathrm{~kg}$. Um alto rendimento de carcaça de cordeiros abatidos aos $25 \mathrm{~kg}$ é obtido ao melhor nível nutricional do nascimento ao abate, e cordeiros abatidos aos 25 ou $33 \mathrm{~kg}$ de peso vivo apresentam carcaças com mesmo estado de engorduramento.

\section{REFERÊNCIAS BIBLIOGRÁFICAS}

BERG, R.T., BUTERFIELD, R.M. Nuevos conceptos sobre desarollo de ganado vacuno. Zaragoza : .Acribia, 1979. 297 .

BERG, R.T., WALTERS, L.E. The meat animal: changes and challenges. Journal Animal Science, v.57, n.2, p.135-146, 1983.

CARVALHO, S. Desempenho, composição corporal e exigências nutricionais de cordeiros machos inteiros, machos castrados e fêmeas alimentadas em confinamento. Santa Maria, 1998. 100p. Dissertação (Mestrado em Zootecnia) - Curso de Pós-graduação em Zootecnia, Universidade Federal de Santa Maria,1998.

DEAMBROSIS, A. Produción y comercialización de carnes. Producuion, crescimiento de carne ovina. Montividéo : Universidade de la Republica, 1972. p.235-236.

HAMMOND, J. Avances en fisiologia zootecnia. Zaragoza , Acribia, 1959. 200p.

KIRTON, H., FOURIE, P.D. JURY,K. E. Growth and development of sheep. III. Growth of the carcass and noncarcass components of the Southdowwn and Romney and their cross and some relationship with composition. New Zeland Journal of Agricultural Research. New Zeland, v.15, p.214-227,1972.

MÜLLER, L. Normas para avaliação de carcaças e concurso de carcaças de novilhos. Santa Maria : Universidade Federal de Santa Maria, Departamento de Zootecnia, 1987. 31p. 
OSÓRIO, J.C.S. Produção de carne ovina. Alternativa para o Rio Grande do Sul. Pelotas : UFPEL, 1998. 166p.

OWEN, J.H. Sheep production. London : Baillière Tindall, 1976. 436p.

ROSA, G.T. Proporções e crescimento de osso, músculo, gordura e componentes não carcaça do peso vivo e crescimento das regiões da carcaça de cordeiros (as) em diferentes métodos de alimentação. Santa Maria- RS 2000. 84p. Dissertação (Mestrados em Zootecnia) - Curso de Pósgraduação em Zootecnia, Universidade Federal de Santa Maria, 2000

SAINZ, D.R. Qualidade das carcaças e da carne ovina e caprina. In: REUNIÃO ANUAL DA SOCIEDADE BRASILEIRA DE ZOOTECNIA, 33, 1996, Fortaleza. Anais... Fortaleza : SBZ, 1996. p.7.

SALOMONI, E. Classificação, tipificação e fatores que influenciam na qualidade da carcaça. Bagé : EMBRAPAUEPAE, 1981. 44p (EMBRAPA-UEPAE, Circular Técnica, 5).

SANTOS, C.L Estudo do desempenho, das características da carcaça e do crescimento alométrico de cordeiros das raças Santa Inês e Bergamácia. Lavras, MG, 1999. 143p. Dissertação (Mestrado em Zootecnia) - Curso de Pós-graduação em Zootecnia, Universidade Federal de Lavras, 1999.

SAS INSTITUTE. Sas User's Guide: Statistics. Version 6. 4 ed. Cary/North Carolina, 1996. V.2. 400p.
SENTS, A.E., WALTERS, L.E., WHITEMAN, J.V. Performance and carcass characterístics of ram lambs slaughtered at different weights. Journal Animal Science, v.55, n.6, p.1360-1368, 1982.

SILVA, C.S. Peso vivo ao abate e características da carcaça de cordeiros Ideal $X$ Ideal, criados em campo nativo com acesso a pastagem cultivada Pelotas, RS, 1986. 92p. Dissertação (Mestrado em Zootecnia) - Faculdade de Agronomia "Eliseu Maciel", Universidade Federal de Pelotas, 1986

SIQUEIRA, E.R. Desempenho e características de carcaça de cordeiros machos e fêmeas da raça Ideal cruzas Texel $\mathbf{x}$ Ideal, criados em pastagem nativa. Pelotas, 1983. 124p. Dissertação (Mestrado em Zootecnia) - Faculdade de Agronomia "Eliseu Maciel", -Universidade Federal de Pelotas, 1983.

SIQUEIRA, E.R., FERNANDES, S. Pesos , rendimentos e perdas da carcaça de cordeiros Corriedale e mestiços Ile de France x Corriedale, terminados em confinamento. Ciência Rural, v.29, n.1, p.143-148,1999.

SOLOMON, M.B., KEMP, J.D., MMODY, W.G., et al. Effect of breed and slaughter weight on physical, chemical and organoleptic properties of lamb carcasses. Journal of Animal Science, v.51, n.2, p.1102-1107,1980.

Ciência Rural, v. 31, n. 6, 2001. 